\title{
NEW DIAGRAM TECHNIQUE FOR PERIODIC ANDERSON MODEL
}

\author{
V. Moskalenko, D. Digor, L. Dogotaru, I. Porcescu \\ Institute of Applied Physics, Academy of Sciences of Moldova, \\ 5 Chişinau,2028, Republic of Moldova
}

(Received July 15, 1996)

\begin{abstract}
A new diagram technique, previously proposed for Hubbard Model, was used for the Periodic Anderson Model (PAM). The Hamiltonian of localized electrons $H_{f}^{0}$ and of free conduction electrons $H_{c}^{0}$ are considered as a main part of the Hamiltonian of PAM and the hybridization term is treated as a perturbation. $H_{f}^{0}$ contains on-site Coulomb interaction and is diagonalized in Hubbard operators. This diagram technique is used for Matsubara Green functions and contains apart from the Wick product of free Green functions new contributions equal to on-site cumulants depending of the Coulomb interaction. If such cumulants are ignored we obtaine the so called Hubbard I approximation for PAM with 3 branches of renormalized energy spectrum of quasiparticles. They are investigated in detail and the chemical potential of the system is determined

Key words: Periodic Anderson model, Coulomb repulsion, conduction electrons, localized electrons, energy gap.
\end{abstract}

PACS number(s): 71.28.+d, 71.27.+a

\section{INTRODUCTION}

The systems with strong electronic correlations, showing unusual thermodynamic, magnetic, transport and superconducting properties are of great interest now. Among them, there are the heavy fermion compounds, the copper oxides, in which high- $T_{c}$ superconductivity was discovered and other materials. Their microscopically quantum - theoretical investigation can be carried out using the periodic Anderson Model (PAM) [1]. We shall discuss the simplest form of this model with the Hamiltonian

$$
\begin{aligned}
& H=H_{c}^{0}+H_{f}^{0}+H_{i n t} \\
& H_{c}^{0}=\sum_{\mathbf{k}, \sigma}(\epsilon(\mathbf{k})-\mu) c_{\mathbf{k} \sigma}^{+} c_{\mathbf{k} \sigma} ; \\
& H_{f}^{0}=\sum_{i, \sigma}\left(E_{f}-\mu\right) f_{i \sigma}^{+} f_{i \sigma}+U \sum_{i} f_{i \uparrow}^{+} f_{i \uparrow} f_{i \downarrow}^{+} f_{i \downarrow} ; \\
& H_{i n t}=\frac{V}{\sqrt{N}} \sum_{i, \mathbf{k}, \sigma}\left[c_{\mathbf{k} \sigma}^{+} f_{i \sigma} e^{i \mathbf{k} \mathbf{R}_{i}}+f_{i \sigma}^{+} c_{\mathbf{k} \sigma} e^{-i \mathbf{k} \mathbf{R}_{i}}\right]
\end{aligned}
$$

were $c_{\mathbf{k} \sigma}^{+}\left(c_{\mathbf{k} \sigma}\right)$ - are creation (annihilation) operators of conduction electrons with wave vector $\mathbf{k}$, energy $\epsilon(\mathbf{k})$, band width $W$ and spin $\sigma ; f_{i \sigma}^{+}\left(f_{i \sigma}\right)$ - the corresponding operators for localized on site $i f$-electrons. $U-$ is on-site Coulomb repulsion of $f$ - electrons, $\mu$-chemical potential of the system. The local $f$ - orbitals with site energy $E_{f}$ are single site hybridized through $V$ with conduction electron states.

The PAM is a rather complicated many-body problem and obtaining some general relations and properties of the renormalized Green's function is useful.

The properties of PAM were discussed in a large num- ber of papers [2-14] where the influence of Coulomb repulsion $U$ and hybridization $V$ on ground state and energy spectrum of quasiparticles of the system, the existence of mixed valence of electrons, the phase transitions of them were investigated and different approaches and approximations were proposed.

In this paper the thermodynamic perturbation theory is developed for the system with Hamiltonian (1.1) supposing that hybridization Hamiltonian is a perturbation. In zero order approximation $f$-electrons are considered localized and Hubbard [15] operators $X_{i}^{n m}$ are used to diagonalize Hamiltonian $H_{f}^{0}$.

$$
\begin{aligned}
c_{i \sigma} & =X_{i}^{0, \sigma}+\sigma X_{i}^{-\sigma, 2} ; \quad f_{i \sigma}^{+}=X_{i}^{\sigma, 0}+\sigma X_{i}^{2,-\sigma} \\
H_{f}^{0} & =\sum_{i, \alpha} E_{\alpha} X_{i}^{\alpha, \alpha}
\end{aligned}
$$

where index $\alpha$ enumerates four on site electron states: without electrons, with one electron having $\sigma$ spin and with two electrons of opposite spins. Their energy are:

$$
E_{0}=0 ; E_{\sigma}=E_{f}-\mu ; E_{2}=U+2\left(E_{f}-\mu\right) .
$$

Conduction electrons in this approximation are considered free and determined by the wave vectors $\mathrm{k}$, spins $\sigma$ and band energy $\epsilon(\mathbf{k})$. The grand partition function of the system in zero approximation is factorized by wave vectors for conduction and by sites $i$ for localized electrons. In such a way the statistical averages with this partition function are calculated for $c$-electrons by making use of Wick theorem for the products of $c$-electron operators. But for the statistical average of localized $f$ electron operator products the generalized Wick theorem proposed in papers [16-18] is employed. This last 
theorem is based on the conception of many particles irreductible Green's functions or Kubo cumulants which appear in realization of these averages.

\section{PERTURBATION THEORY}

The evolution operator of the thermodynamic perturbation theory is

$$
\begin{aligned}
U(\beta) & =T \exp \left(-\int_{0}^{\beta} H_{\text {int }}(\tau) d \tau\right) \\
& =\sum_{n=0}^{\infty} \frac{(-1)^{n}}{n !} T\left(H_{\text {int }}\left(\tau_{1}\right) \ldots H_{\text {int }}\left(\tau_{n}\right)\right) .
\end{aligned}
$$

The free energy of the system is equal:

$$
\begin{aligned}
& F=F_{0}-\frac{1}{\beta} \ln \langle U(\beta)\rangle_{0} \\
& F_{0}=-\frac{1}{\beta} \ln \operatorname{Tr}\left\{e^{-\beta\left(H_{c}^{0}+H_{f}^{0}\right)}\right\} .
\end{aligned}
$$

The average $\langle U(\beta)\rangle_{0}$ can be obtained taking into account only the even $n=2 m$ degrees of perturbation theory because of the structure of $H_{\text {int }}$ multiplies. Then in such a product of $2 m$ multiplies there are in total $4 m$ operators. Only the operator structures which have m operators $c, \bar{c}, f, \bar{f}$ each kind must be considered in the future because other operator combinations have zero statistical averages. The number of such structures is equal to $C_{2 m}^{m}$ and changing the indices $i, \mathbf{k}, \sigma$ of summing and of $\tau$ integration, we can prove that all of them are equivalent. Taking one of such operator structures and multiplying it by $C_{2 m}^{m}$ after dividing by $(2 m)$ ! we obtain

$$
\begin{aligned}
\langle U(\beta)\rangle_{0} & =1+\sum_{m=1}^{\infty} \frac{V^{2 m}}{N^{m}(m !)^{2}} \sum_{i_{1} \mathbf{k}_{1} \sigma_{1}} \ldots \sum_{i_{2 m}, \mathbf{k}_{2 m}, \sigma_{2 m}} \int d \tau_{1 \ldots} \int d \tau_{2 m} \\
& \times\left\langle T \overline{\mathbf{c}}_{\mathbf{k}_{1} \sigma_{1}}\left(\tau_{1}\right) \ldots \overline{\mathbf{k}}_{\mathbf{k}_{m} \sigma_{m}}\left(\tau_{m}\right) c_{\mathbf{k}_{m+1} \sigma_{m+1}}\left(\tau_{m+1}\right) \ldots c_{\mathbf{k}_{2 m} \sigma_{2 m}}\left(\tau_{2 m}\right)\right\rangle_{0} \\
& \times\left\langle T f_{i_{1} \sigma_{1}}\left(\tau_{1}\right) \ldots f_{i_{m} \sigma_{m}}\left(\tau_{m}\right) \bar{f}_{i_{m+1}}\left(\tau_{m+1}\right) \ldots \bar{f}_{i_{2 m} \sigma_{2 m}}\left(\tau_{2 m}\right)\right\rangle_{0} \\
& \times \exp \left\{i\left(\mathbf{k}_{1} \mathbf{R}_{1}+\ldots+\mathbf{k}_{m} \mathbf{R}_{m}-\mathbf{k}_{m+1} \mathbf{R}_{m+1}-\ldots-\mathbf{k}_{2 m} \mathbf{R}_{2 m}\right)\right\}
\end{aligned}
$$

The statistical average of conduction electron operators calculated by using Wick theorem gives us the sum of $m$ ! member each of them equal to the product of $m$ free conducting electron propagators $G^{c(0)}$.

By changing all the above mentioned, indices we can prove that all of them are equivalent and m! additional multiplier appears in the numerator of $(2.3)$.

So we have

$$
\begin{aligned}
\langle U(\beta)\rangle_{0} & =1+\sum_{m=1}^{\infty} \frac{V^{2 m}}{N^{m} m !} \sum_{i_{1} \mathbf{k}_{1} \sigma_{1}} \ldots \sum_{i_{2 m}, \mathbf{k}_{2 m}, \sigma_{2 m}} \int d \tau_{1 \ldots} \int d \tau_{2 m} \\
& \times\left\langle T \bar{c}_{\mathbf{k}_{1} \sigma_{1}}\left(\tau_{1}\right) c_{\mathbf{k}_{2 m} \sigma_{2 m}}\left(\tau_{2 m}\right)\right\rangle_{0} \ldots\left\langle T \bar{c}_{\mathbf{k}_{m} \sigma_{m}}\left(\tau_{m}\right) c_{\mathbf{k}_{m+1} \sigma_{m+1}}\left(\tau_{m+1}\right)\right\rangle_{0} \\
& \times\left\langle T f_{i_{1} \sigma_{1}}\left(\tau_{1}\right) \ldots f_{i_{m} \sigma_{m}}\left(\tau_{m}\right) \bar{f}_{i_{m+1} \sigma_{m+1}}\left(\tau_{m+1}\right) \ldots \bar{f}_{i_{2 m}\left(\sigma_{2 m}\right)}\left(\tau_{2 m}\right)\right\rangle_{0} \\
& \times \exp \left\{i\left(\mathbf{k}_{1} \mathbf{R}_{1}+\ldots+\mathbf{k}_{m} \mathbf{R}_{m}-\mathbf{k}_{m+1} \mathbf{R}_{m+1}-\ldots-\mathbf{k}_{2 m} \mathbf{R}_{2 m}\right)\right\}
\end{aligned}
$$

where

$$
G^{c(0)}\left(\mathbf{k}_{1} \sigma_{1} \tau_{1} \mid \mathbf{k}_{2} \sigma_{2} \tau_{2}\right)=-\left\langle T c_{\mathbf{k}_{1} \sigma_{1}}\left(\tau_{1}\right) \bar{c}_{\mathbf{k}_{2} \sigma_{2}}\left(\tau_{2}\right)\right\rangle_{0} .
$$

Equation (2.4) has the form of the perturbation theory for Hubbard model if the hopping of the $f$-electrons of this last model is considered as a perturbation:

$$
H_{\text {int }}=-\sum_{i j \sigma} t(j-i) f_{j \sigma}^{+} f_{i \sigma}
$$


In (2.4) the instantaneous matrix elements $t(i-j)$ of Hubbard model are replaced by the dynamicall propagators $G^{c(0)}$ multiplied by $V^{2}$

In the next the generalized Wick theorem of [16-18] is used. For $m=1$ the average $\left\langle f_{1} \bar{f}_{2}\right\rangle_{0}$ is free propagator for localized and strong interacting $f$-electrons

$$
G^{f(0)}\left(\mathbf{k}_{1} \sigma_{1} \tau_{1} \mid \mathbf{k}_{2} \sigma_{2} \tau_{2}\right)=-\left\langle T f_{\mathbf{k}_{1} \sigma_{1}}\left(\tau_{1}\right) \bar{f}_{\mathbf{k}_{2} \sigma_{2}}\left(\tau_{2}\right)\right\rangle_{0}
$$

For $m=2$ the average is equal [16-18]

$$
\begin{aligned}
\left\langle T f_{1} f_{2} \bar{f}_{3} \bar{f}_{4}\right\rangle_{0} & =\left\langle T f_{1} \bar{f}_{4}\right\rangle_{0}\left\langle T f_{2} \bar{f}_{3}\right\rangle_{0} \\
& -\left\langle T f_{1} \bar{f}_{3}\right\rangle_{0}\left\langle T f_{2} \bar{f}_{4}\right\rangle_{0}+\langle 12 \mid \overline{3} \overline{4}\rangle_{0}^{i r}
\end{aligned}
$$

where $1=\left(i_{1}, \sigma_{1}, \tau_{1}\right)$

$$
\begin{aligned}
& \langle 12 \mid \overline{3} \overline{4}\rangle_{0}^{i r}=G_{2}^{(0) i r}\left(i_{1} \sigma_{1} \tau_{1}, i_{2} \sigma_{2} \tau_{2} \mid i_{3} \sigma_{3} \tau_{3}, i_{4} \sigma_{4} \tau_{4}\right) \\
& =\delta_{i_{1} i_{2}} \delta_{i_{1} i_{3}} \delta_{i_{1} i_{4}} G_{2}^{(0) i r}\left(\sigma_{1} \tau_{1}, \sigma_{2} \tau_{2} \mid \sigma_{3} \tau_{3}, \sigma_{4} \tau_{4}\right) \\
& G_{2}^{(0) i r}\left(\sigma_{1} \tau_{1}, \sigma_{2} \tau_{2} \mid \sigma_{3} \tau_{3}, \sigma_{4} \tau_{4}\right) \\
& =\left\langle T f_{\sigma_{1}}\left(\tau_{1}\right) f_{\sigma_{2}}\left(\tau_{2}\right) \bar{f}_{\sigma_{3}}\left(\tau_{3}\right) \bar{f}_{\sigma_{4}}\left(\tau_{4}\right)\right\rangle_{0}-\left\langle T f_{\sigma_{1}}\left(\tau_{1}\right) \bar{f}_{\sigma_{4}}\left(\tau_{4}\right)\right\rangle_{0} \\
& \times\left\langle T f_{\sigma_{2}}\left(\tau_{2}\right) \bar{f}_{\sigma_{3}}\left(\tau_{3}\right)\right\rangle_{0}+\left\langle T f_{\sigma_{1}}\left(\tau_{1}\right) \bar{f}_{\sigma_{3}}\left(\tau_{3}\right)\right\rangle_{0}
\end{aligned}
$$

$$
\left\langle T f_{\sigma_{1}}\left(\tau_{1}\right) \bar{f}_{\sigma_{4}}\left(\tau_{4}\right)\right\rangle_{0}
$$

All the quantities on the right hand of equation (2.5) are one-site ones. They depend of spin indices and $\tau$ variables and are independent of site indices. $G_{2}^{(0) i r}$ is the two particles irreducible Green function or the Kubo cumulant. When Coulomb interaction is zero such functions do not exist.

The first two components of (2.5) are of Wick type but the last one is a new contribution conditioned by strong electronic correlations. For $m=3$ the existing statistical average $\left\langle T f_{1} f_{2} f_{3} \bar{f}_{4} \bar{f}_{5} \bar{f}_{6}\right\rangle_{0}$ contains 3 ! Wick contributions each of them equal to the product of three propagators $G^{f(0)}$. Then there are 9 contributions each of them equal to the product of propagator $G^{f(0)}$ and one irreductible two particles Green's function and there is also one contribution equal to three particles irreductible Green's function $G_{3}^{(0) i r}$.
In the general case of statistical average of $2 m f$ operators the generalized Wick theorem gives us $m$ ! components of Wick type, each of them equal to the product $m$ propagators, and then there are the sum of products of different kind of Kubo cumulants, organized in such a way that the number of particles in all these cumulants is equal to $m$. The sign of all these contributions is determined by the number of permutations of fermi $f$-operators which is necessary to obtain the given cumulant structure.

Some of vacuum diagrams are shown on fig. 1 .

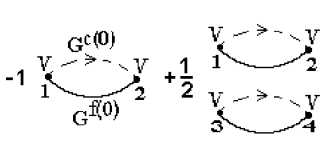

a)

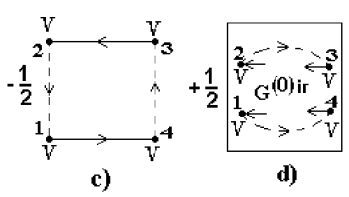

Fig. 1.
Here the thin directed full line is the $G^{f(0)}$ propagator and the directed dotted line is $G^{c(0)}$ one. The point of the diagram contains hybridization $V$. 1.a diagram is the simplest connected one but 1.b diagram is a deconnected one. More complicated connected diagrams are 1.d,1.c ones. The analysis of the vacuum diagram's structure gives us the equation

$$
\langle U(\beta)\rangle_{0}=1+\sum_{n=1}^{\infty} \frac{\left[\langle U(\beta)\rangle_{0}^{c}\right]^{n}}{n !}=\exp \left[\langle U(\beta)\rangle_{0}^{c}\right]
$$

where $\langle U(\beta)\rangle_{0}^{c}$ is the connected part of all the vacuum diagrams. This equation is a very well known theorem of connected diagrams of statistical physics.

\section{ONE PARTICLE GREEN'S FUNCTIONS}

We shall discuss now the renormaslized $f$-electron one particle Green's function:

$$
G^{f f}\left(\mathbf{x} \sigma \tau \mid \mathbf{x}^{\prime} \sigma^{\prime} \tau^{\prime}\right)=-\left\langle T f_{\mathbf{x} \sigma}(\tau) \bar{f}_{\mathbf{x}^{\prime} \sigma^{\prime}}\left(\tau^{\prime}\right) U(\beta)\right\rangle_{0}^{c}
$$

where the index $c$ points out that only connected propagator diagrams must be considered.

Equation (3.1) can be transformed by using the arguments of the previous section. In such a way we obtain

$$
\begin{aligned}
& G^{f f}\left(x \mid x^{\prime}\right)=G^{f(0)}\left(x \mid x^{\prime}\right)-\sum_{m=1}^{\infty} \frac{V^{2 m}}{N^{m} m !} \int d \tau_{1} \ldots \int d \tau_{2 m} \sum_{i_{1} \mathbf{k}_{1} \sigma_{1}} \ldots \\
& \ldots \sum_{i_{2 m} \mathbf{k}_{2 m} \sigma_{2 m}}\left\langle T \bar{c}_{1} c_{2 m}\right\rangle_{0} * \ldots\left\langle T \bar{c}_{m} c_{m+1}\right\rangle_{0} *\left\langle T f_{x} \bar{f}_{x^{\prime}} f_{1} \ldots\right. \\
& \left.\ldots f_{m} \bar{f}_{m+1} \ldots \bar{f}_{2 m}\right\rangle_{0} \exp \left\{i\left(\mathbf{k}_{1} \mathbf{R}_{1}+\ldots+\mathbf{k}_{m} \mathbf{R}_{m}-\mathbf{k}_{m+1} \mathbf{R}_{m+1}-\ldots-\mathbf{k}_{2 m} \mathbf{R}_{2 m}\right)\right\} .
\end{aligned}
$$


Here $x$ and $x^{\prime}$ indices of $f$-operators stand for $\mathrm{x} \sigma \tau$ and $\mathbf{x}^{\prime} \sigma^{\prime} \tau^{\prime}$ correspondingly. The other indices of $f$-operators are of the same kind, for example $1=i_{1} \sigma_{1} \tau_{1}$ but for $c$-electron operators we have $1=\mathbf{k}_{1} \sigma_{1} \tau_{1}$ and so on. The existence of the exponents gives us a possibility to use also local presentation for $c$-electron quantities or wave vector presentation for $f$-operators.

If in the last statistical average of (3.2) we take into account only Wick type contributions, we shall obtain the chain type diagrams shown in fig. 2.

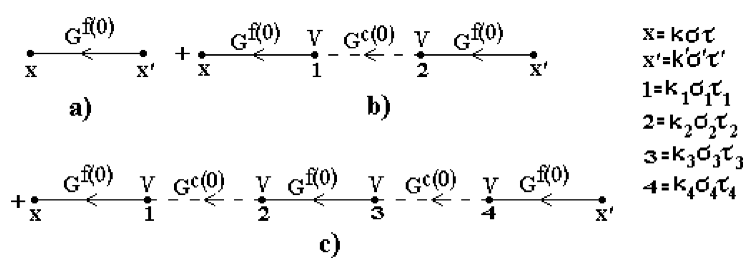

Fig. 2.

Here all the irreducible Green's functions were omitted. All these diagrams are weak by connected because they can be devided into two parts by cutting one line. They can be summed using Fourier-presentation. We obtain Hubbard I approximation for Dyson equation:

$$
\begin{gathered}
G_{\sigma}^{f f}\left(\mathbf{k} \mid i \omega_{n}\right)=G_{\sigma}^{f(0)}\left(i \omega_{n}\right)\left(1+V^{2} G^{c(0)}\left(\mathbf{k} \mid i \omega_{n}\right) G_{\sigma}^{f f}\left(\mathbf{k} \mid i \omega_{n}\right)\right) \\
G_{\sigma}^{f f}\left(\mathbf{k} \mid i \omega_{n}\right)=\left[G_{\sigma}^{f(0)}\left(i \omega_{n}\right)^{-1}-V^{2} G^{c(0)}\left(\mathbf{k} \mid i \omega_{n}\right)\right]^{-1}
\end{gathered}
$$

In this approximation it is easy to obtain also the Dyson equation for renormalized conduction electron Green's function $G_{\sigma}^{c c}\left(\mathbf{k} \mid i \omega_{n}\right)$. Free electron propagators are

$$
\begin{gathered}
G_{\sigma}^{c c(0)}\left(\mathbf{k} \mid i \omega_{n}\right)=\left(i \omega_{n}-\epsilon_{0}\right)^{-1} ; \\
\epsilon_{0}=\epsilon(\mathbf{k})-\mu ; \\
G_{\sigma}^{f(0)}\left(i \omega_{n}\right)=\frac{1-\bar{n}_{-\sigma}}{i \omega_{n}-\epsilon_{1}}+\frac{\bar{n}_{-\sigma}}{i \omega_{n}-\epsilon_{2}} ; \\
\epsilon_{1}=E_{f}-\mu ; \epsilon_{2}=U+E_{f}-\mu ; \\
\bar{n}=\left\langle\bar{f}_{\sigma} f_{\sigma}\right\rangle_{0}=\left\{\exp \left(-\beta \epsilon_{1}\right)+\exp \left[-\beta\left(\epsilon_{1}+\epsilon_{2}\right)\right]\right\} /\{1 \\
\left.+2 \exp \left(-\beta \epsilon_{1}\right)+\exp \left[-\beta\left(\epsilon_{1+} \epsilon_{2}\right)\right]\right\} .
\end{gathered}
$$

The energy spectrum of quasiparticles is determined by equation:

$$
\left(E-\epsilon_{0}\right)-V^{2}\left[(1-\bar{n}) /\left(E-\epsilon_{1}\right)+\bar{n} /\left(E-\epsilon_{2}\right)\right]=0 .
$$

For large values of Coulomb interaction $U \rightarrow \infty$ three energetic branches are:

$$
\begin{aligned}
& E_{1,2}(\mathbf{k})=\left[\epsilon_{0}+\epsilon_{1} \pm \sqrt{\left(\epsilon_{0}-\epsilon_{1}\right)^{2}+4 V^{2}(1-\bar{n})}\right] / 2 \\
& +\frac{V^{2} \bar{n}}{2 \epsilon_{2}}\left[1 \pm\left(\epsilon_{0}-\epsilon_{1}\right) / \sqrt{\left(\epsilon_{0}-\epsilon_{1}\right)^{2}+4 V^{2}(1-\bar{n})}\right] \\
& +o\left(1 / U^{2}\right) ; \\
& E_{3}(\mathbf{k})=\epsilon_{2}+V^{2} \bar{n} / \epsilon_{2}+o\left(1 / U^{2}\right) .
\end{aligned}
$$

The chemical potential of the system with $N_{e}$ ellectrons is determined from

$(1 / \beta) \sum_{\omega_{n}} \sum_{\mathbf{k} \sigma}\left[G_{\sigma}^{f f}\left(\mathbf{k} \mid i \omega_{n}\right)+G_{\sigma}^{c c}\left(\mathbf{k} \mid i \omega_{n}\right)\right] \exp \left(i \omega_{n} 0^{+}\right)=N_{e}$

This equation is reduced to

$$
\begin{aligned}
& \sum_{\sigma} \sum_{\mathbf{k}}\left\{A_{\sigma}(\mathbf{k}) /\left[\exp \left(\beta E_{1 \sigma}\right)+1\right]+B_{\sigma}(\mathbf{k}) /\left[\exp \left(\beta E_{2 \sigma}+1\right]\right.\right. \\
+ & \left.C_{\sigma}(\mathbf{k}) /\left[\exp \left(\beta E_{3 \sigma}\right)+1\right]\right\}=N_{e}
\end{aligned}
$$

where in the limit of large $U$ value $U \rightarrow \infty$ these coefficients are

$$
A_{\sigma}(\mathbf{k}) \approx 1-\bar{n} v^{2}(\mathbf{k}) ; B_{\sigma} \approx 1-\bar{n} u^{2}(\mathbf{k}) ; C_{\sigma}(\mathbf{k}) \approx \bar{n}
$$

where

$$
\begin{aligned}
& u^{2}(\mathbf{k})=\frac{1}{2}\left[1+\left(\epsilon_{0}-\epsilon_{1}\right) / \sqrt{\left(\epsilon_{0}-\epsilon_{1}\right)^{2}+4 V^{2}(1-\bar{n})}\right] \\
& v^{2}(\mathbf{k})=\left[1-\left(\epsilon_{0}-\epsilon_{1}\right) / \sqrt{\left(\epsilon_{0}-\epsilon_{1}\right)^{2}+4 V^{2}(1-\bar{n})}\right] / 2 .
\end{aligned}
$$

In this limit there are two subbands with an energy gap between them. The sum of the widths of these two subbands is equal to the width of the bare conduction band but the distance between the bottom of the lower subband and the top of the upper subband is equal to plus the width of energy gap.

The dependence of the electron's number on the chemical potential of the system was obtained. For example, in the case when the chemical potential is placed into the lower subband this dependence is

$$
\frac{N_{e}}{2 N}=\frac{(\mu+W / 2)}{W}+\frac{V^{2}(1-\bar{n})}{\left(E_{f}-\mu\right) W}
$$




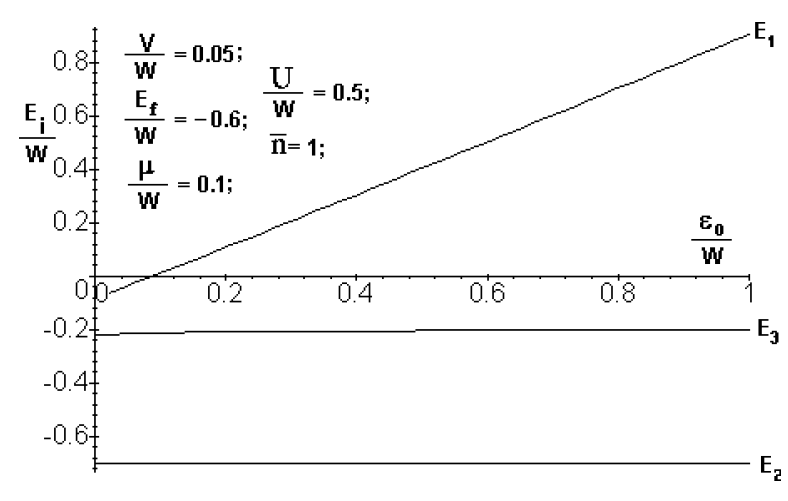

Fig. 3.

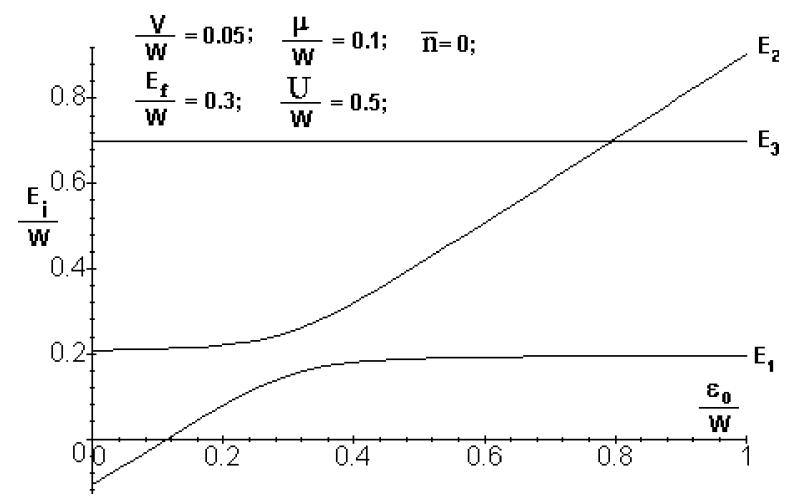

Fig. 4.

Because the local initial level $E_{f}$ is located in the energy gap of this renormalized system in our case of temperature equal to zero $E_{f}-\mu>0$ and the average $\bar{n}=0$. When $\mu$ is more higher than the top of the upper subband $N_{e}=3 N$.

The calculated values of the renormalized quasiparticle energies as function of $\epsilon_{0} / W$ for $\mathrm{T}=0$ and different values of the main parameters of the theory are shown in figures $3-6$.

The origin of the energy scale is at the bottom of the bare conduction electron band, and the conduction-band limits are 0 and $W$, for the bottom and the top, respectively. $U$ is considered positive.

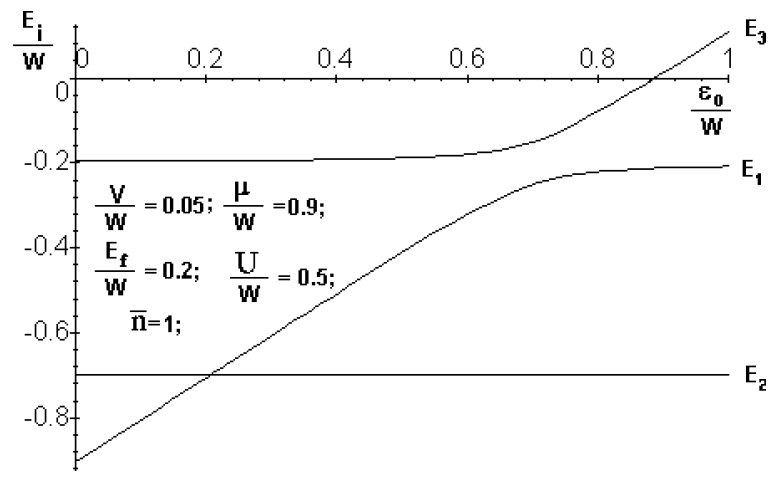

Fig. 5.

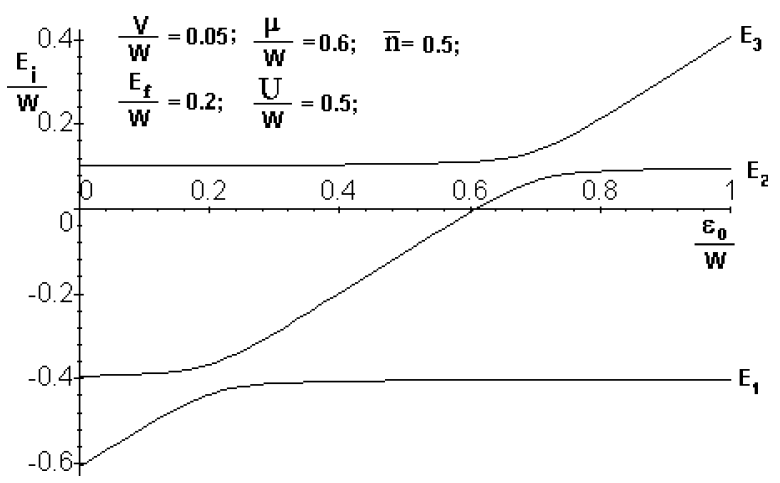

Fig. 6.

The bare energy band of conduction electrons can remain unsplit by hybridization energy (fig. 3). It can be split into two subbands (fig. 4-5.) or in general case into three subbands (fig. 6).

The first situation is realized when one of the following inequalities takes place:

a) $E_{f}+U<0$; b) $E_{f}>W$; c) $W-U<E_{f}<0$;

The appearance of two subbands is realized on condition that:

$0<\mu<E_{f}$ or $E_{f}+U<\mu<W$

and the last situation takes place when

$$
0<E_{f}<\mu<E_{f}+U<W
$$

[1] P. W. Anderson, Phys. Rev. 124, 41 (1961).

[2] H. I. Leder, B. Mühlschlegel, Z. Physik B 29, 341 (1978).

[3] P. Coleman, Phys. Rev. B 29, 3035 (1984).

[4] J. Callway et al., Phys. Rev. B 38, 2583 (1988).

[5] J. Callway, L. Tan, H. Zheng, Phys. Rev. B 50, 1369 (1994).

[6] T. M. Rice, K. Ueda, Phys. Rev. Lett. 55, 995 (1985).

[7] R. Blankenbecler, J. R. Fulco, W. Gill, D. I. Scalapino, Phys. Rev. Lett. 58, 411 (1987).
[8] K. Yamoto, K. Ueda, Jur. Phys. Soc. Japan 59, 3284 (1990)

[9] K. Yamada and K. Yosida, Electron correlation and magnetism in narrow-band systems, ed. T. Morya (Berlin Springer, 1981, p. 210).

[10] B. Möller, P. Wölfle, Phys. Rev. B 48, 10320 (1993).

[11] E. Halvorsen, Q. Czycholl, Jour. Phys. Condens. Matter. 8, 1775 (1996)

[12] A. Kuzemsky, International Centre For Theoretical 
Physics IC $/ 93 / 336$.

[13] A. Kuzemsky, J. C. Parlebas, H. Bec, Physica A 198, 606 (1993); A. Kuzemsky, Rapid Communication JINR Dubna, 3/60/1993.

[14] B. H. Brandow, Phys. Rev. B 33, 215 (1986).

[15] J. Hubbard, Proc. Roy. Soc. A 276, 233 (1963).
[16] M. I. Vladimir, V. A. Moskalenko, Teor. Matem. Fiz. 82, 428 (1990).

[17] S. I. Vacaru, M. I. Vladimir, V. A. Moskalenko, Teor. Matem. Fiz. 85, 248 (1990).

[18] N. N. Bogoliubov, V. A. Moskalenko, Dokl. Akad. Nauk SSSR 136, 1107 (1991).

\section{НОВА ДІАГРАМНА ТЕХНІКА ДЛЯ ПЕРІОДИЧНОЇ МОДЕЛІ АНДЕРСОНА}

В. Москаленко, Д. Дігор, Л. Доготару, І. Порческу

Інститут прикладної фізики Академіӥ наук Молдови, 5 Кішінеу, 2028, Республіка Молдова

Нова діаграмна техніка, яка була попередньо запропонована для моделі Габбарда, використана для періодичної моделі Андерсона. Гамільтоніани локалізованих електронів $H_{f}^{0}$ та вільних електронів провідності $H_{c}^{0}$ вважаються основною частиною гамільтоніана періодичної моделі Андерсона, а гібридизаційний член розглядають як збурення. $H_{f}^{0}$ включає спонтанну взаємодію Кулона та діагоналізується в операторах Габбарда. Запропонована діаграмна техніка використовується для мацубарівських функцій Гріна та містить, поряд із добутком Віка вільних функцій Гріна, нові внески, рівні кумулянтам на вузлах, що залежать від кулонівської взаємодії. При нехтуванні останніми отримуємо так зване наближення Габбарда I для періодичної моделі Андерсона з трьома гілками ренормалізованого енергетичного спектра квазічастинок. Представлено іх детальний аналіз, а також визначено хемічний потенціял системи. 